\section{Summary}

Prognostic Aspects of Meningomyeloceles

All possible complications due to meningomyeloceles are reviewed and the longterm prognosis discussed. In particular, the paper examines changes both in therapies available and in attitudes of the medical profession during the last years towards this disease.

\section{Bibliographie}

[1] Gross, R. E., The Surgery of Infancy and Childhood, W. B. Saunders, 1953.

[2] Hayden, P. W., Shurtleff, D. B., et Broy, A. B., Custody of the myelodysplastic child. Implications for selection for early treatment, Pediatrics 53, 253-256 (1974).

[3] Laurence, $K . M$., et Tew, B. J., Follow-up of 65 survivors from the 425 cases of spina bifida born in south Wales between 1965 and 1952, Develop. Med. Child Neurol. Suppl. 13, 1-13 (1967).

[4] Lorber, J., Results of treatment of myelomeningocele. An analysis of 524 unselected cases, with special reference to possible selection for treatment, Develop. Med. Child Neurol. 13, 279 (1971).

[5] Lorber, J., et Shloss, A. L., The adolescent with myelomeningocele, Develop. Med. Child Neurol. Suppl. 29, 113 (1973).

[6] Nash, D. F., et Ellison, D., The impact of total care with special reference to myelodysplasie, Develop. Med. Child Neurol. Suppl. 22, 1-11 (1970).

[7] Points of view: Non-treatment of defective babies, Lancet ii, 1123 (1979), Lancet i, 156 (1980).

[8] Sharrard, W. J. W., Zachary, R. B., et Lorber, J., Survival and paralysis in open myelomeningoceles with special reference to time of repair of the spinal lesion, Develop. Med. Child Neurol. Suppl. 13, 35-50 (1967).

[9] Tew, B. J., et Laurence, $K . M$., Mothers, brothers and sisters of patients with spina bifida, Develop. Med. Child Neurol. Suppl. 29, 69 (1973).

[10] Tew, B. J., et Laurence, K. M., The effect of admission to hospital and Surgery on children with spina bifida, Develop. Med. Child Neurol. Suppl. 37, 119-125 (1976).

\title{
Das Alpha-1-Fetoprotein-Screening aus der Sicht der Gesundheitsbehörden ${ }^{1}$
}

\author{
PD Dr. med.J.-C. Vuille, Schularztamt der Stadt Bern, 3011 Bern
}

Es wird die These vertreten, dass es nicht möglich ist, aus Erwägungen über die gesellschaftliche «Bürde» ein wesentliches öffentliches Interesse am Alpha-1-Fetoprotein-Screening abzuleiten. Aus den von der IVKommission des Kantons Bern freundlicherweise zur Verfügung gestellten Daten über die gesamten Aufwendungen für 9 zufällig ausgewählte MMC-Kinder mit Behinderungen verschiedenen Schweregrades lässt sich abschätzen, dass für Behandlung, Hilfsmittel und Sonderschulung sämtlicher MMC-Kinder im Kanton Bern von allen Kostenträgern zusammen jährlich rund $1-1 \frac{1}{2}$ Mio. Franken aufgewendet werden, was im Vergleich mit anderen Investitionen im öffentlichen Bereich von untergeordneter Bedeutung sein dürfte. Immerhin wäre auch eine Einsparung von dieser Grössenordnung nicht zu verachten, wenn sie realisiert werden könnte. Es ist aber nicht anzunehmen, dass der «Wegfall» von etwa 5 mehrfach behinderten Kindern pro Jahr in Spitälern, Sonderschulen und anderen Einrichtungen der Behindertenhilfe zu wirklich spürbaren Entlastungen führen würde, da sich der Minderbedarf an Dienstleistungen auf sehr viele verschiedene Personen und Institutionen verteilen würde.

Im Bereich der Heilpädagogik wird das Hauptproblem der achtziger und neunziger Jahre nicht der Mangel an Institutionen und Personal sein, sondern der «Mangel» an behinderten Kindern. Viele der bestehenden Institutionen werden entweder überhaupt nicht mehr oder nicht mehr rationell geführt werden können. Wohl wird bei der notwendig werdenden Strukturreform auch auf die Verminderung der Anzahl MMC-Kinder Rücksicht zu nehmen sein; da aber spezifisches therapeutisches «Know-how für die wenigen MMC-Kinder, die es immer geben wird, stets vorhanden sein muss, kann die blosse Verminderung der Anzahl von den Gesundheitsbehörden nicht nur positiv beurteilt werden. Jedenfalls werden die Probleme für die Behörden nicht im selben Masse kleiner, wie die Zahl der MMCKinder abnimmt.

Auch ohne exakte Kosten-Nutzen-analyse kann somit festgehalten werden, dass kein überwiegendes öffentliches Interesse am pränatalen Screening geltend gemacht werden kann. Die Indikation muss sich auf Erwägungen über die Bürde für den betroffenen Menschen und seine Familie abstützen. Diese Bürde kann im Einzelfall so gross werden, dass schwere irreparable Schäden für die ganze Familie entstehen. Auf der anderen Seite gibt es belastbare Familien, für die auch ein schwer behindertes Kind keine wesentliche Beeinträchtigung der Lebensqualität bedeutet. Das Hauptproblem dürfte darin liegen, dass es für die Eltern selber und für Aussenstehende unmöglich ist, in der Frühschwangerschaft eine Prognose über die Belastbarkeit 10, 20 oder 30 Jahre später zu stellen.

${ }^{1}$ Zusammenfassung und Schlussfolgerungen. 